\title{
Muonium hydride: The lowest density crystal
}

\author{
Youssef Kora and Massimo Boninsegni $\odot^{*}$ \\ Department of Physics, University of Alberta, Edmonton, Alberta, Canada T6G 2E1 \\ Dam Thanh Son \\ Kadanoff Center for Theoretical Physics, The University of Chicago, Chicago, Illinois 60637, USA \\ Shiwei Zhang \\ Center for Computational Quantum Physics, Flatiron Institute, New York, New York 10010, USA \\ and Department of Physics, The College of William and Mary, Williamsburg, Virginia 23187, USA
}

(Received 13 March 2021; accepted 23 April 2021; published 10 May 2021)

\begin{abstract}
A muonium hydride molecule is a bound state of muonium and hydrogen atoms. It has half the mass of a parahydrogen molecule and very similar electronic properties in its ground state. The phase diagram of an assembly of such particles is investigated by first-principles quantum simulations. In the bulk limit, the lowtemperature equilibrium phase is a crystal of extraordinarily low density, lower than that of any other known atomic or molecular crystal. Despite the low density and particle mass, the melting temperature is surprisingly high (close to $9 \mathrm{~K}$ ). No (metastable) supersolid phase is observed. We investigated the physical properties of nanoscale clusters (up to 200 particles) of muonium hydride and found the superfluid response to be greatly enhanced compared to that of parahydrogen clusters. The possible experimental realization of these systems is discussed.
\end{abstract}

DOI: 10.1103/PhysRevResearch.3.023113

\section{INTRODUCTION}

An intriguing open question in condensed matter physics, one with potential practical significance, is whether there exists a lower bound for the density of a crystal. In other words, how large can the average distance be between nearest-neighbor atoms, before crystalline long-range order is suppressed by thermal and quantum fluctuations? It remains unclear whether a lower limit exists, or how to approach it experimentally. We begin our discussion by rehashing a few basic facts about the crystalline phase of matter.

Crystallization occurs at low temperatures $(T)$ in almost all known substances, as the state of lowest energy (ground state) is approached. Classically, the ground state is one in which the potential energy of the interaction among the constituent particles is minimized, a condition that corresponds to an orderly arrangement of particles in regular, periodic lattices. In most cases, quantum mechanics affects this fundamental conclusion only quantitatively, as the zero-point motion of particles results in lower equilibrium densities and melting temperatures, with respect to what one would predict classically; typically, these corrections are relatively small. Only in

\footnotetext{
*m.boninsegni@ualberta.ca

Published by the American Physical Society under the terms of the Creative Commons Attribution 4.0 International license. Further distribution of this work must maintain attribution to the author(s) and the published article's title, journal citation, and DOI.
}

helium is the classical picture upended by quantum mechanics, as the fluid resists crystallization all the way down to a temperature $T=0 \mathrm{~K}$, under the pressure of its own vapor.

In many condensed matter systems, the interaction among atoms is dominated by pairwise contributions, whose ubiquitous features are a strong repulsion at interparticle distances less than a characteristic length $\sigma$, as well as an attractive part, which can be described by an effective energy well depth $\epsilon$. The dimensionless parameter $\Lambda=\hbar^{2} /\left[m \epsilon \sigma^{2}\right]$, where $m$ is the particle mass, quantifies the relative importance of quantummechanical effects in the ground state of the system.

It has been established for a specific model pair potential incorporating the above basic features, namely the LennardJones potential, and for Bose statistics, that the equilibrium phase is a crystal if $\Lambda<\Lambda_{c} \approx 0.15$; it is a (super)fluid if $0.15 \lesssim \Lambda \lesssim 0.46$, while, for $\Lambda>0.46$, the system only exists in the gas phase [1]. The value of $\Lambda_{c}$ is estimated [2] to be slightly higher $(\approx 20 \%)$ for systems obeying Fermi statistics. For substances whose elementary constituents are relatively simple atoms or molecules, this result provides a useful, general criterion to assess their propensity to crystallize, as measured by their proximity in parameter space to a quantum phase transition to a fluid phase. It can be used to infer, at least semiquantitatively, macroscopic properties of the crystal, such as its density and melting temperature, both of which generally decrease [1] as $\Lambda \rightarrow \Lambda_{c}$.

The two stable isotopes of helium have the highest known values of $\Lambda$, namely $0.24(0.18)$ for ${ }^{3} \mathrm{He}\left({ }^{4} \mathrm{He}\right)$; for all other naturally occurring substances, $\Lambda$ is considerably lower. The next highest value is that of molecular hydrogen $\left(\mathrm{H}_{2}\right)$, namely 
$\Lambda=0.08$, quickly decreasing for heavier elements and compounds. At low temperatures, $\mathrm{H}_{2}$ forms one of the least dense crystals known, of density $\rho=0.0261 \AA^{-3}$ (mass density $0.086 \mathrm{~g} / \mathrm{cc}$ ). The low mass of a $\mathrm{H}_{2}$ molecule (half of that of a ${ }^{4} \mathrm{He}$ atom) and its bosonic character (its total spin is $S=0$ in its ground state) led to the speculation that liquid $\mathrm{H}_{2}$ may turn into a superfluid at low temperatures [3], just as ${ }^{4} \mathrm{He}$. In practice, no superfluid phase is observed (not even a metastable one), as molecular interactions, and specifically [4] the relatively large value of $\sigma(\sim 3 \AA)$, cause $\mathrm{H}_{2}$ to crystallize at a temperature $T=13.8 \mathrm{~K}$.

The present consensus is that $\mathrm{H}_{2}$ is a nonsuperfluid, insulating crystal at low temperatures, including in reduced dimensions [5,6]; only small clusters of parahydrogen $(\sim 30$ molecules or less) are predicted [7-10] to turn into a superfluid at $T \sim 1 \mathrm{~K}$, for which some experimental evidence has been reported [11]. If, hypothetically, the mass of the molecules could be progressively reduced, while leaving the interaction unchanged, thus increasing $\Lambda$ from its value for $\mathrm{H}_{2}$ all the way to $\Lambda_{c}$, several intriguing scenarios might arise, including a low-temperature superfluid liquid phase, freezing into a low-density crystal at $T=0$, and even a supersolid phase, namely one enjoying at the same time crystalline order and superfluidity [12]. Obviously, the value of $\Lambda$ can also be modified by changing one or both of the interaction parameters $(\epsilon$ and $\sigma)$ independently of the mass; in this work, however, we focus for simplicity on the effect of mass on the physics of the system.

One potential way to tune the mass is via the substitution of protons or electrons with muons. For example, an assembly of molecules of muonium hydride ( $\mathrm{HMu}$ ) differs from $\mathrm{H}_{2}$ by the replacement of one of the two protons with an antimuon $\left(\mu^{+}\right)$, whose mass is approximately $11 \%$ of that of a proton. Quantum chemistry calculations have shown that it is very similar in size to $\mathrm{H}_{2}$, and has the same quantum numbers in the ground state [13]. It is therefore not inconceivable that the interaction between two HMu might be quantitatively close to that between two $\mathrm{H}_{2}$ (we further discuss this aspect below). In this case the value of the parameter $\Lambda$ is $\sim 0.14$, i.e., very close to $\Lambda_{c}$ for a Bose system. This leads to the speculation that this substance may crystallize into a highly quantal solid, displaying strikingly unique behavior, compared to ordinary crystals. In order to investigate such a scenario, and more specifically to gain insight into the effect of mass reduction, we studied theoretically the low-temperature phase diagram of a hypothetical condensed phase of $\mathrm{HMu}$, by means of first-principles computer simulations of a microscopic model derived from that of $\mathrm{H}_{2}$.

The main result is that the equilibrium phase of the system at low temperature is a crystal of very low density, some $\sim 5 \%$ lower than the $T=0$ equilibrium density of liquid ${ }^{4} \mathrm{He}$. Despite the low density, however, such a crystal melts at a fairly high temperature, close to $9 \mathrm{~K}$, i.e., only a few $\mathrm{K}$ lower than that of $\mathrm{H}_{2}$. No superfluid phases are observed, either fluid or crystalline, as exchanges of indistinguishable particles, known to underlie superfluidity [14], are strongly suppressed in this system, much as they are in $\mathrm{H}_{2}$, by the relatively large size of the hard-core repulsion at short distances (i.e., $\sigma$ ). As a result, the behavior of this hypothetical system can be largely understood along classical lines. This underscores once again the crucial role of exchanges of identical particles in destabilizing the classical picture, which is not qualitatively altered by the zero-point motion of particles alone [15]; it also reinforces the conclusion [4] reached elsewhere that it is the size of the hard-core diameter of the intermolecular interaction that prevents superfluidity in $\mathrm{H}_{2}$, not the depth $\epsilon$ of its attractive part.

To complement our investigation, we also studied nanoscale HMu clusters of varying sizes, comprising up to few hundred molecules. We find the behavior of these clusters to be much closer to that of ${ }^{4} \mathrm{He}$ (rather than $\mathrm{H}_{2}$ ) clusters. For example, at $T=1 \mathrm{~K}$ the structure of HMu clusters is liquidlike, and their superfluid response approaches $100 \%$, even for the largest cluster considered (200 HMu molecules). Thus, while mass reduction does not bring about substantial physical differences between the behavior of bulk $\mathrm{HMu}$ and that of $\mathrm{H}_{2}$, it significantly differentiates the physics of nanoscale size clusters.

\section{MODEL}

We model the HMu molecules as pointlike, identical particles of mass $m$ and spin $S=0$, thus obeying Bose statistics. For the bulk studies, the system is enclosed in a cubic cell of volume $V=L^{3}$ with periodic boundary conditions in the three directions, giving a density of $\rho=N / V$. For the cluster studies, the $N$ particles are placed in a supercell of large enough size to remove the boundary effects. The quantum-mechanical many-body Hamiltonian reads as follows,

$$
\hat{H}=-\lambda \sum_{i} \nabla_{i}^{2}+\sum_{i<j} v\left(r_{i j}\right),
$$

where the first (second) sum runs over all particles (pairs of particles), $\lambda \equiv \hbar^{2} / 2 m=21.63 \mathrm{~K} \AA^{2}$ (reflecting the replacement of a proton with a $\mu^{+}$in a $\mathrm{H}_{2}$ molecule), $r_{i j} \equiv\left|\mathbf{r}_{i}-\mathbf{r}_{j}\right|$, and $v(r)$ denotes the pairwise interaction between two $\mathrm{HMu}$ molecules, which is assumed spherically symmetric.

In order to decide on an adequate model potential to adopt in our calculation, we use as our starting point the $\mathrm{H}_{2}$ intermolecular potential, for which a considerable amount of theoretical work has been carried out [16-18]. We consider here for definiteness the ab initio pair potential proposed in Ref. [18]. The most important effect of the replacement of one of the protons of the $\mathrm{H}_{2}$ molecule with a $\mu^{+}$is the shift of the center of mass of the molecule away from the midpoint, and toward the proton. We assume that this effect provides the leading-order correction with respect to the $\mathrm{H}_{2}$ intermolecular potential, and we set out to obtain a corrected version of the interaction for the new geometry, as illustrated in Fig. 1. We use the program provided in Ref. [18] to generate a potential as a function of the distance between the midpoints and the angular configurations, and then transform that to a potential as a function of the distance between the centers of mass and the angular configurations. Finally, we average over the angular configurations to obtain a one-dimensional isotropic potential. We take the distance between the proton and the $\mu^{+}$ to be that computed in Ref. [19], which differs only slightly from the distance between the two protons in the $\mathrm{H}_{2}$ molecule.

In Fig. 2, we compare the potential energy of interaction between two $\mathrm{HMu}$ molecules resulting from this procedure 


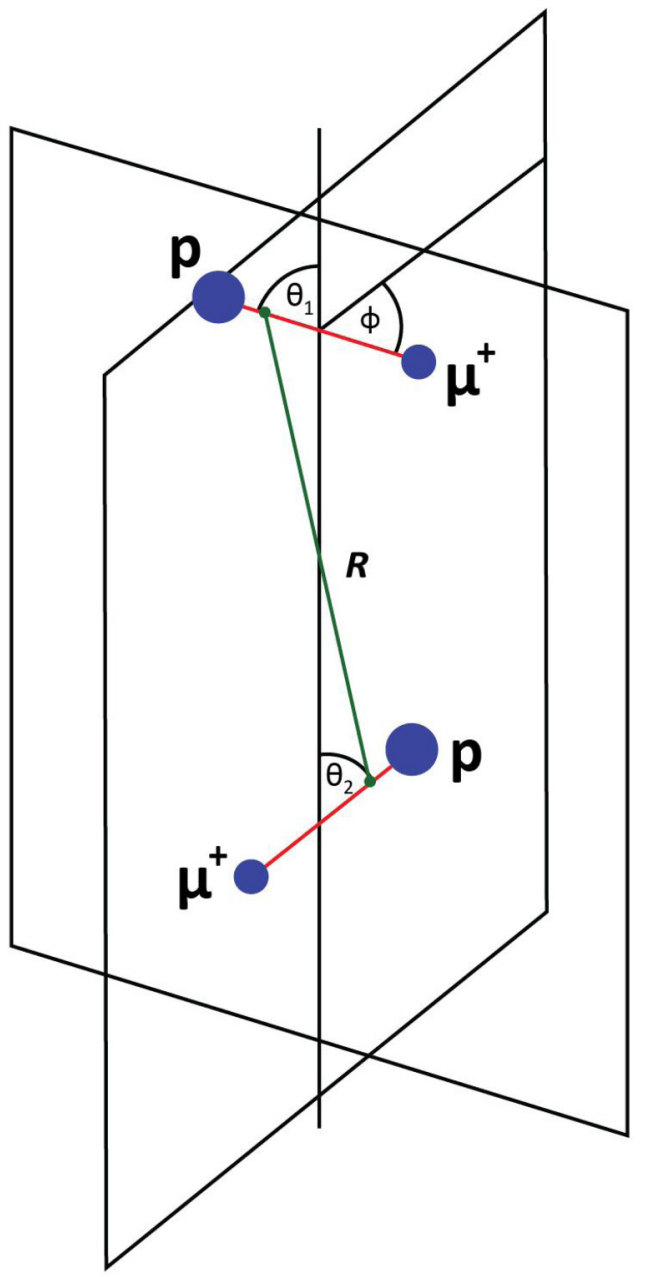

FIG. 1. Geometry utilized in the calculation of the effective interaction between two HMu molecules. Shown is the line connecting the centers of mass of the two molecules, as well the three angles describing their relative orientation, i.e., the two polar angles $\theta_{1}, \theta_{2}$, as well as the azimuthal angle $\phi$.

with that obtained for two $\mathrm{H}_{2}$ molecules, i.e., with the center of mass at the midpoint. The comparison suggests that the displacement of the center of mass of the molecule results in a slight stiffening of the potential at a short distance. Indeed, in the range of average interparticle separations explored in this work (namely the 3.5-3.7 A range), the differences between the interactions are minimal. Also shown in Fig. 2 is the Silvera-Goldman potential [16], which is arguably the most widely adopted in theoretical studies of the condensed phase of $\mathrm{H}_{2}$, and has proven to afford a quantitatively accurate [20] description of the structure and energetics of the crystal. As one can see, it has a significantly smaller diameter and is considerably "softer" than the potential of Ref. [18]; the reason is that it incorporates, in an effective way, nonadditive contributions (chiefly triplets), whose overall effect is to soften the repulsive core of the pairwise part computed ab initio.

It therefore seems reasonable to utilize the SilveraGoldman potential [16] to carry out our study, as the use of a quantitatively more accurate potential is not likely to affect the conclusions of our study in a significant way. Furthermore, because the majority of the theoretical studies of condensed

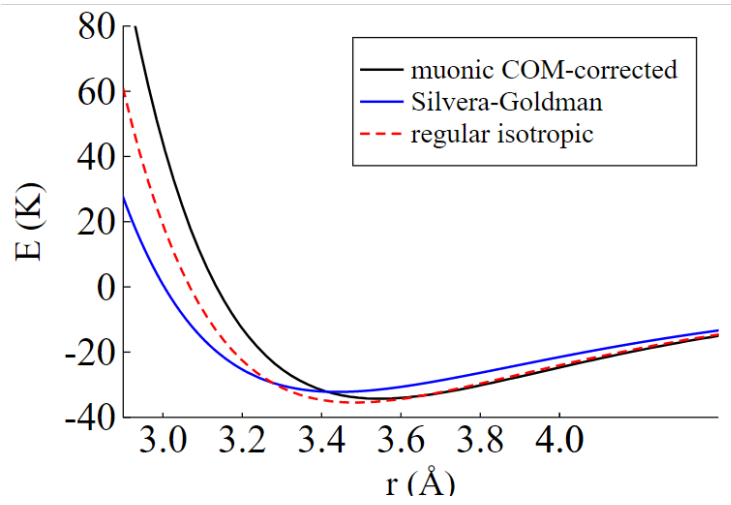

FIG. 2. The intermolecular interaction energy $E$ (in $\mathrm{K}$ ) as a function of distance $(\AA)$ between the centers of mass of the molecules, for the $\mathrm{H}_{2}$ (red, dashed) and HMu (black, solid line) cases, obtained with the aid of the programs provided in Ref. [18]. Also shown (blue, solid line) is the Silvera-Goldman potential.

$\mathrm{H}_{2}$ have been carried out using the Silvera-Goldman potential, its use in this study allows us to assess the effect of mass alone.

\section{METHODOLOGY}

The low-temperature phase diagram of the thermodynamic system described by Eq. (1) as a function of density and temperature has been studied in this work by means of firstprinciples numerical simulations, based on the continuousspace worm algorithm [21,22]. Since this technique is by now fairly well established, and extensively described in the literature, we shall not review it here; we used a variant of the algorithm in which the number of particles $N$ is fixed $[8,9]$. Details of the simulation are standard; we made use of the fourth-order approximation for the short imaginary-time $(\tau)$ propagator (see, for instance, Ref. [23]), and all of the results presented here are extrapolated to the $\tau \rightarrow 0$ limit. We generally found numerical estimates for structural and energetic properties of interest here, obtained with a value of the time step $\tau \sim 3.0 \times 10^{-3} \mathrm{~K}^{-1}$ to be indistinguishable from the extrapolated ones, within the statistical uncertainties of the calculation. We carried out simulations of systems typically comprising a number $N$ of particles. In the cluster calculations, $N$ can be chosen arbitrarily. For the bulk, the precise value of $N$ depends on the type of crystalline structure assumed; typically, $N$ was set to 128 for simulations of bodycentered-cubic (bcc) and face-centered-cubic (fcc) structures, and 216 for hexagonal-close-packed (hcp). However, we also performed a few targeted simulations with twice as many particles, in order to gauge the quantitative importance of finite-size effects.

Physical quantities of interest for the bulk calculations include the energy per particle and pressure as a function of density and temperature, i.e., the thermodynamic equation of state in the low-temperature limit. We estimated the contribution to the energy and the pressure arising from pairs of particles at distances greater than the largest distance allowed by the size of the simulation cell (i.e., $L / 2$ ), by approximating the pair-correlation function $g(r)$ with 1 , for $r>L / 2$. We have also computed the pair-correlation function and the 


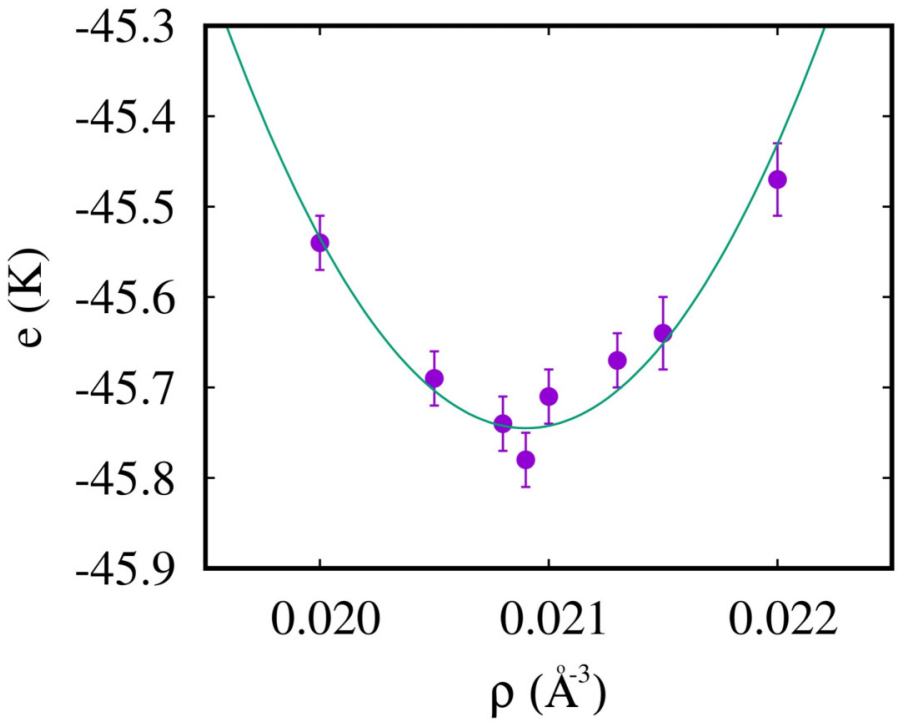

FIG. 3. Energy per particle $e$ in $(\mathrm{K})$ as a function of density $\rho$ in $\AA^{-3}$, computed at temperature $T=1 \mathrm{~K}$. The solid line is a quadratic fit to the data. These energies are computed assuming a bcc solid structure.

related static structure factor, in order to assess the presence of crystalline order, which can also be detected through visual inspection of the imaginary-time paths.

We probed for possible superfluid order through the direct calculation of the superfluid fraction using the wellestablished winding number estimator [24]. In order to assess the propensity of the system to develop a superfluid response, and its proximity to a superfluid transition, we also rely on a more indirect criterion, namely we monitor the frequency of cycles of permutations of identical particles involving a significant fraction of the particles in the system. While there is no quantitative connection between permutation cycles and the superfluid fraction [25], a global superfluid phase requires exchanges of macroscopic numbers of particles (see, for instance, Ref. [14]).

\section{RESULTS}

\section{A. Bulk}

We simulated crystalline phases of HMu assuming different structures, namely bcc, fcc, and hcp. At low temperatures, all of these crystals remain stable in the simulation. The energy difference between different crystal structures is typically small, of the order of the statistical errors of our calculation, i.e., few tenths of a K. This is similar to what is observed in $\mathrm{H}_{2}$ [20]). Consequently, we did not attempt to establish what the actual equilibrium structure is, as this is not central to our investigation.

As a general remark, we note that the estimates of the physical quantities in which we are interested remain unchanged below a temperature $T=2 \mathrm{~K}$. Thus, results for temperatures lower than $2 \mathrm{~K}$ can be considered ground state estimates.

We begin by discussing the equation of state of the system in the $T \rightarrow 0$ limit. Figure 3 shows the energy per $\mathrm{HMu}$ molecule computed as a function of density for a

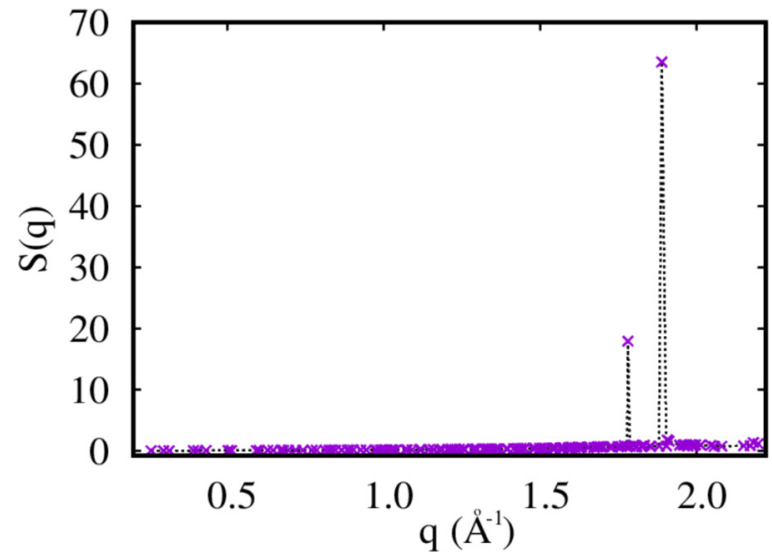

FIG. 4. Static structure factor computed at temperature $T=1 \mathrm{~K}$, for a hcp crystal of HMu of density $\rho=0.0209 \AA^{-3}$. The simulated system comprises $N=216$ molecules.

temperature $T=1 \mathrm{~K}$. The solid line is a quadratic fit to the data, whose fitting parameters yield the equilibrium density $\rho_{0}=0.02090(5) \AA^{-3}$, corresponding to an average intermolecular distance $3.63 \AA$, as well as the ground state energy $e_{0}=-45.75(2) \mathrm{K}$. The ground state energy is almost exactly [20] one-half of that of $\mathrm{H}_{2}$, with a kinetic energy of 70.4(1) K, virtually identical to that of $\mathrm{H}_{2}$ at equilibrium, i.e., at a $30 \%$ higher density.

The equilibrium density $\rho_{0}$ is lower than that of superfluid ${ }^{4} \mathrm{He}$, which is $0.02183 \AA^{-3}$. The system is in the crystalline phase, however, as we can ascertain through the calculation of the static structure factor $S(q)$, shown in Fig. 4. The result shown in the figure pertains to a case in which particles are initially arranged into a hcp crystal. The sharp peaks occurring at values of $q$ corresponding to wave vectors in the reciprocal lattice signal long-range crystalline order.

It is interesting to compare the structure of a $\mathrm{HMu}$ crystal to that of two other reference quantum solids, namely $\mathrm{H}_{2}$ (more precisely, para- $\mathrm{H}_{2}$ ) at its equilibrium density, namely $\rho=0.0261 \AA^{-3}$, and solid ${ }^{4} \mathrm{He}$ near melting, at density $\rho=0.0287 \AA^{-3}$. The pair-correlation functions shown in Fig. 5 were computed for hcp crystals at temperature $T=1$ $\mathrm{K}$ (the results for $\mathrm{H}_{2}$ and ${ }^{4} \mathrm{He}$ were obtained in Ref. [26]). While the fact that the peaks appear at different distances reflects the difference in density, ${ }^{4} \mathrm{He}$ being the most and $\mathrm{HMu}$ the least dense crystals, the most noticeable feature is that the height of the peaks is much more pronounced in $\mathrm{H}_{2}$ than in $\mathrm{HMu}$ and ${ }^{4} \mathrm{He}$, whose peak heights and widths are similar.

Figure 6 shows the pressure of a HMu crystal computed at $T=1 \mathrm{~K}$, as a function of density. In the relatively narrow density range considered, a linear fit is satisfactory, and allows us to compute the speed of sound $v=(m \rho \kappa)^{-1 / 2}$, where $\kappa=$ $\rho^{-1}(\partial \rho / \partial P)$ is the compressibility. We obtained the speed of sound $1300 \pm 100 \mathrm{~m} / \mathrm{s}$ at the equilibrium density.

Next, we discuss the possible superfluid properties of the crystal, as well as of the fluid into which the crystal melts upon raising the temperature. There is no evidence that the crystalline phase of $\mathrm{HMu}$ may display a finite superfluid response in the $T \rightarrow 0$ limit. Indeed, the observation is that the behavior of this crystal is virtually identical to that of solid $\mathrm{H}_{2}$, as far as superfluidity is concerned. The main 


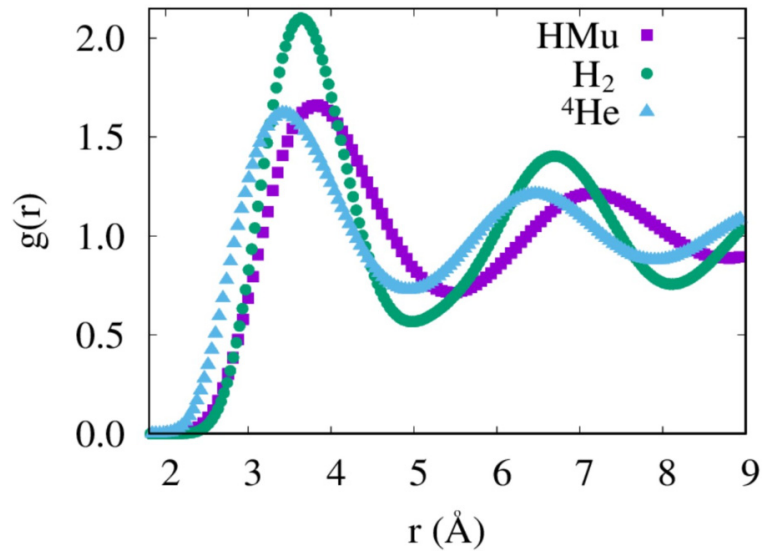

FIG. 5. Pair-correlation function $g(r)$ for $\mathrm{HMu}$ at density $\rho_{0}=$ $0.0209 \AA^{-3}$ and $T=1 \mathrm{~K}$ (squares). Also shown for comparison are the corresponding correlation functions for para- $\mathrm{H}_{2}$ at $\rho=$ $0.0261 \AA^{-3}$ (circles), and solid ${ }^{4} \mathrm{He}$ at $\rho=0.0287 \AA^{-3}$ (triangles), at the same temperature. In all three cases the crystalline structure is hcp. Statistical errors are smaller than symbol sizes.

observation is that exchanges of identical particles, which underlie superfluidity, are strongly suppressed in $\mathrm{HMu}$, much as they are in $\mathrm{H}_{2}$, mainly due to the relatively large diameter of the repulsive core of the pairwise interaction. Indeed, exchanges are essentially nonexistent in solid $\mathrm{HMu}$ at the lowest temperature considered here $(T=1 \mathrm{~K})$, much as in solid $\mathrm{H}_{2}$; the reduction of particle mass by a factor of 2 does not quantitatively alter the physics of the system in this regard.

As temperature is raised, we estimate the melting temperature to be around $T \sim 9 \mathrm{~K}$. We arrive at that conclusion by computing the pressure as a function of temperature for different densities (at and below $\rho_{0}$ ), and by verifying that the system retains solid order even at negative pressure, all the way up to $T=8 \mathrm{~K}$. No evidence is seen of any metastable, underpressurized fluid phase; on lowering the density, the system eventually breaks down in solid clusters, just as in $\mathrm{H}_{2}$ [5]. Above $8 \mathrm{~K}$, the pressure of the solid phase jumps and stable fluid phases appear at lower densities, signaling the

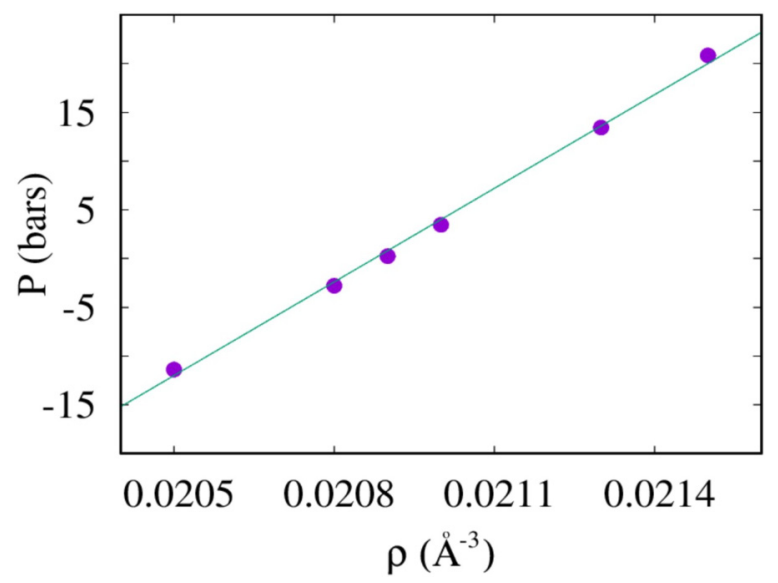

FIG. 6. Pressure of the HMu bcc crystal as a function of density at $T=1 \mathrm{~K}$ (circles). The line is a linear fit to the data. Statistical errors are smaller than symbol sizes. occurrence of melting. These fluid phases do not display any superfluid properties; exchanges of two or three particles occur with a frequency of approximately $0.1 \%$. It is known that quantum-mechanical effects in $\mathrm{H}_{2}$ contribute about one-half of the Lindemann ratio at melting [26]; we did not perform the same calculation for this system, but in this system quantum mechanics should be more important, on account of the lighter mass. However, qualitatively melting appears to occur very similarly as in $\mathrm{H}_{2}$. Also, our results suggest that thermal expansion, which is negligible [27] in solid $\mathrm{H}_{2}$, is likely very small in this crystal.

\section{B. Clusters}

It is also interesting to study the physics of nanoscale size clusters of $\mathrm{HMu}$, and compare their behavior to that of parahydrogen clusters, for which, as mentioned above, superfluid behavior is predicted at temperatures of the order of $1 \mathrm{~K}$, if their size is approximately 30 molecules or less. Crystalline behavior emerges rather rapidly for parahydrogen clusters of greater size [28], with "supersolid" behavior occurring for specific clusters [29]. This calculation is carried out with the same methodology adopted for the bulk phase of the system, the only difference being that the simulation cell is now taken large enough that a single cluster forms. In this respect, the behavior of $\mathrm{HMu}$ clusters is closer to that of parahydrogen than ${ }^{4} \mathrm{He}$ clusters, in that no external potential is required to keep them together (as in the case of ${ }^{4} \mathrm{He}$ ), at least at sufficiently low temperature $(\lesssim 4 \mathrm{~K})$.

However, the reduced molecular mass makes the physics of HMu clusters both quantitatively and qualitatively different from that of parahydrogen clusters. The first observation is that the superfluid response is greatly enhanced; specifically, it is found that clusters with as many as $N=200$ molecules are close to $100 \%$ superfluid at $T=1 \mathrm{~K}$, and remain at least $50 \%$ superfluid up to $T \lesssim 4 \mathrm{~K}$, at which point they begin to evaporate, i.e., they do not appear to stay together as normal clusters. This is in stark contrast with parahydrogen clusters, where exchanges are rare in clusters of more than 30 molecules at this temperature, and they would involve at most $\sim 10$ particles. Consistently, the superfluid response is insignificant in parahydrogen clusters, and they stay together almost exclusively due to the potential energy of the interaction. Our results highlight the importance of the energetic contribution of quantum-mechanical exchanges in the stabilization of $\mathrm{HMu}$ clusters at low temperatures. Indeed, at $T=1 \mathrm{~K}$ we observe cycles of exchanges involving all of the particles in $\mathrm{HMu}$ clusters comprising as many as 200 molecules.

Figure 7 shows density profiles computed with respect to the center of mass of the system for three different clusters of $\mathrm{HMu}$, comprising $N=30,100$, and 200 molecules. As mentioned above, all these clusters are essentially fully superfluidic at this temperature. These density profiles are qualitatively similar, nearly featureless, and reminiscent of those computed for ${ }^{4} \mathrm{He}$ droplets [30]. The comparison with the density profile computed for a cluster of 30 parahydrogen molecules, also shown in Fig. 7, illustrates how the latter is much more compact and displays pronounced oscillations, which are indicative of a well-defined, solidlike shell structure. 


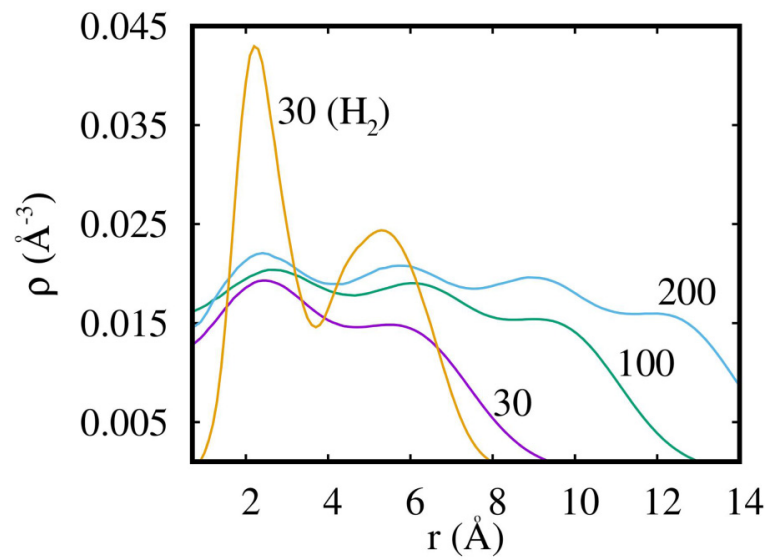

FIG. 7. Radial density profiles (computed with respect to the center of mass) for HMu clusters comprising $N=30,100$, and 200 molecules, at a temperature $T=1 \mathrm{~K}$. At this temperature, all of these clusters are essentially $100 \%$ superfluid. Also shown for comparison is the same quantity for a cluster of $N=30$ parahydrogen molecules, at the same temperature; this cluster has no significant superfluid response.

As the number of molecules increases, clusters of $\mathrm{HMu}$ ought to evolve into solidlike objects, their structure approaching that of the bulk. The calculation of the radial superfluid density $[25,31]$ suggests that crystallization begins to occur at the center of the cluster; for example, at $T=1 \mathrm{~K}$ the largest HMu cluster studied here $(N=200)$ displays a suppressed superfluid response inside a central core of approximately $5 \AA$ radius, in which crystalline order slowly begins to emerge, while the rest of the cluster is essentially entirely superfluid. We expect the nonsuperfluid core to grow with the size of the cluster. In other words, large clusters consist of a rigid, insulating core and a superfluid surface layer, with a rather clear demarcation between the two. This is qualitatively different from the behavior observed in parahydrogen clusters, in which crystallization occurs for much smaller sizes, and "supersolid" clusters simultaneously displaying solidlike and superfluid properties can be identified [29].

\section{CONCLUSIONS}

We have investigated the low-temperature phase diagram of a bulk assembly of muonium hydride molecules, by means of first-principles quantum simulations. Our model assumes a pairwise, central interaction among $\mathrm{HMu}$ molecules which is identical to that of $\mathrm{H}_{2}$ molecules. By a mapping of the $\mathrm{H}_{2}$ interatomic potentials derived from $a b$ initio calculations, we showed that this model provides a reasonable description of the $\mathrm{HMu}-\mathrm{HMu}$ interation. It is certainly possible to carry out a more accurate determination of the pair potential, but the main effect of the lower $\mu^{+}$mass should be that of increasing the diameter of the repulsive core of the interaction, in turn suppressing exchanges even more. As illustrated in Ref. [15], the exchanges of identical particles play a crucial role in destabilizing the classical picture in a many-body system; when exchanges are suppressed, quantum zero-point motion can only alter such a picture quantitatively, not qualitatively. Alternatively, this can be understood by the fact that $\Lambda$ is decreased as the hard-core radius is increased, making the system more classical. Obviously, regarding the interaction as spherically symmetric is also an approximation, but one that affords quantitatively accurate results for parahydrogen [20], for which the potential energy of interaction among molecules plays a quantitatively more important role in shaping the phase diagram of the condensed system.

Perhaps the most significant observation of this study is the different physics of bulk and nanoscale size clusters of $\mathrm{HMu}$. Bulk $\mathrm{HMu}$ is very similar to $\mathrm{H}_{2}$; despite its very low density (lower than that of superfluid ${ }^{4} \mathrm{He}$ ), the equilibrium crystalline phase is stable below a temperature of about $9 \mathrm{~K}$, much closer to the melting temperature of $\mathrm{H}_{2}(13.8 \mathrm{~K})$ than the mass difference may have led one to expect. No evidence is observed of any superfluid phase, neither liquid nor crystalline, underscoring once again how, in order for a supersolid phase to be possible in continuous space, it requires some physical mechanism to cause a "softening" of the repulsive part of the pair potential at short distances [32], even if only along one direction, as in the case of a dipolar interaction [33]. On the other hand, clusters of HMu including up to a few hundred molecules display superfluid behavior similar to that of ${ }^{4} \mathrm{He}$ clusters. This suggests that, as the value of the quantumness parameter $\Lambda$ approaches $\Lambda_{c}$ from below, one may observe nanoscale superfluidity in clusters of rather large size.

We conclude by discussing the possible experimental realization of the system described in this work. The replacement of elementary constituents of matter, typically electrons, with other subatomic particles of the same charge, such as muons [34], has been discussed for a long time, and some experimental success has been reported. Even a bold scenario consisting of replacing all electrons [35] in atoms with muons (the so-called "muonic matter") has been considered; recently, a long-lived "pionic helium" has been created [36]. Thus, it also seems plausible to replace a proton in a $\mathrm{H}_{2}$ molecule with an antimuon; indeed, muonium chemistry has been an active area of research for several decades [37]. In order for "muonium condensed matter" to be feasible, a main challenge to overcome is the very short lifetime of the $\mu^{+}$, of the order of a $\mu \mathrm{s}$.

\section{ACKNOWLEDGMENTS}

This work was supported by the Natural Sciences and Engineering Research Council of Canada, a Simons Investigator grant (D.T.S.) and the Simons Collaboration on Ultra-Quantum Matter, which is a grant from the Simons Foundation (651440, D.T.S.). Computing support of Compute Canada and of the Flatiron Institute is gratefully acknowledged. The Flatiron Institute is a division of the Simons Foundation. 
[1] Y. Kora, M. Boninsegni, D. T. Son, and S. Zhang, Tuning the quantunmness of simple Bose systems: A universal phase diagram, Proc. Natl. Acad. Sci. USA 117, 27231 (2020).

[2] L. H. Nosanow, L. J. Parish, and F. J. Pinski, Zero-temperature properties of matter and the quantum theorem of corresponding states: The liquid-to-crystal phase transition for Fermi and Bose systems, Phys. Rev. B 11, 191 (1975).

[3] V. L. Ginzburg and A. A. Sobyanin, Can liquid molecularhydrogen be superfluid? JETP Lett. 15, 242 (1972).

[4] M. Boninsegni, Search for superfluidity in supercooled liquid parahydrogen, Phys. Rev. B 97, 054517 (2018).

[5] M. Boninsegni, Low-temperature phase diagram of condensed para-hydrogen in two dimensions, Phys. Rev. B 70, 193411 (2004).

[6] M. Boninsegni, Ground State Phase Diagram of Parahydrogen in One Dimension, Phys. Rev. Lett. 111, 235303 (2013).

[7] P. Sindzingre, D. M. Ceperley, and M. L. Klein, Superfluidity in Clusters of $p-\mathrm{H}_{2}$ Molecules, Phys. Rev. Lett. 67, 1871 (1991).

[8] F. Mezzacapo and M. Boninsegni, Superfluidity and Quantum Melting of $p-\mathrm{H}_{2}$ Clusters, Phys. Rev. Lett. 97, 045301 (2006).

[9] F. Mezzacapo and M. Boninsegni, Structure, superfluidity, and quantum melting of hydrogen clusters, Phys. Rev. A 75, 033201 (2007).

[10] M. Boninsegni, Superfluid response of parahydrogen clusters in superfluid ${ }^{4}$ He, J. Low Temp. Phys. 201, 193 (2020).

[11] S. Grebenev, B. Sartakov, J. P. Toennies, and A. F. Vilesov, Evidence for superfluidity in para-hydrogen clusters inside helium-4 droplets at 0.15 Kelvin, Science 289, 1532 (2000).

[12] M. Boninsegni and N. V. Prokof'ev, Supersolids: What and where are they? Rev. Mod. Phys. 84, 759 (2012).

[13] Specifically, the average distance between the proton and the muon in a $\mathrm{HMu}$ molecule is estimated at $0.8 \AA$, whereas that between the two protons in a $\mathrm{H}_{2}$ molecule is $0.74 \AA$. See Refs. $[19,38]$.

[14] R. P. Feynman, Atomic theory of the $\lambda$ transition in helium, Phys. Rev. 91, 1291 (1953).

[15] M. Boninsegni, L. Pollet, N. Prokof'ev, and B. Svistunov, Role of Bose Statistics in Crystallization and Quantum Jamming, Phys. Rev. Lett. 109, 025302 (2012).

[16] I. F. Silvera and V. V. Goldman, The isotropic intermolecular potential for $\mathrm{H}_{2}$ and $\mathrm{D}_{2}$ in the solid and gas phases, J. Chem. Phys. 69, 4209 (1978).

[17] P. Diep and J. K. Johnson, An accurate $\mathrm{H}_{2}-\mathrm{H}_{2}$ interaction potential from first principles, J. Chem. Phys. 112, 4465 (2000).

[18] K. Patkowski, W. Cencek, P. Jankowski, K. Szalewicz, J. B. Mehl, G. Garberoglio, and A. H. Harvey, Potential energy surface for interactions between two hydrogen molecules, J. Chem. Phys. 129, 094304 (2008).

[19] B.-L. Zhou, J.-M. Zhu, and Z.-C. Yan, Variational calculation of muonium hydride, J. Phys. B: At., Mol. Opt. Phys. 38, 305 (2005).
[20] F. Operetto and F. Pederiva, Diffusion Monte Carlo study of the equation of state of solid para- $\mathrm{H}_{2}$, Phys. Rev. B 73, 184124 (2006).

[21] M. Boninsegni, N. Prokof'ev, and B. Svistunov, Worm Algorithm for Continuous-Space Path Integral Monte Carlo Simulations, Phys. Rev. Lett. 96, 070601 (2006).

[22] M. Boninsegni, N. V. Prokof'ev, and B. V. Svistunov, Worm algorithm and diagrammatic Monte Carlo: A new approach to continuous-space path integral Monte Carlo simulations, Phys. Rev. E 74, 036701 (2006).

[23] M. Boninsegni, Permutation sampling in path integral Monte Carlo, J. Low Temp. Phys. 141, 27 (2005).

[24] E. L. Pollock and D. M. Ceperley, Path-integral computation of superfluid densities, Phys. Rev. B 36, 8343 (1987).

[25] F. Mezzacapo and M. Boninsegni, Local Superfluidity of Parahydrogen Clusters, Phys. Rev. Lett. 100, 145301 (2008).

[26] M. Dusseault and M. Boninsegni, Atomic displacements in quantum crystals, Phys. Rev. B 95, 104518 (2017).

[27] F. Fernandez-Alonso, C. Cabrillo, R. Fernández-Perea, F. J. Bermejo, M. A. González, C. Mondelli, and E. Farhi, Solid para-hydrogen as the paradigmatic quantum crystal: Three observables probed by ultrahigh-resolution neutron spectroscopy, Phys. Rev. B 86, 144524 (2012).

[28] F. Mezzacapo and M. Boninsegni, Classical and quantum physics of hydrogen clusters, J. Phys.: Condens. Matter 21, 164205 (2009).

[29] F. Mezzacapo and M. Boninsegni, On the possible "supersolid" character of parahydrogen clusters, J. Phys. Chem. A 115, 6831 (2011).

[30] P. Sindzingre, M. L. Klein, and D. M. Ceperley, Path-Integral Monte Carlo Study of Low-Temperature ${ }^{4} \mathrm{He}$ Clusters, Phys. Rev. Lett. 63, 1601 (1989).

[31] Y. Kwon, F. Paesani, and K. B. Whaley, Local superfluidity in inhomogeneous quantum fluids, Phys. Rev. B 74, 174522 (2006).

[32] M. Boninsegni, Supersolid phases of cold atom assemblies, J. Low Temp. Phys. 168, 137 (2012).

[33] Y. Kora and M. Boninsegni, Patterned supersolids in dipolar Bose systems, J. Low Temp. Phys. 197, 337 (2019).

[34] P. O. Egan, Muonic helium, in Atomic Physics 7, edited by D. Kleppner and F. M. Pipkin (Plenum, New York, 1981), p. 373.

[35] J. A. Wheeler, Nanosecond matter, in Energy in Physics, War and Peace: A Festschrift Celebrating Edward Teller's 80th Birthday, edited by H. Mark and L. Wood (Kluwer, Dordrecht, 1988), Chap. 10, pp. 266-290.

[36] H. Masaki, H. Aghai-Khozani, A. Sótér, A. Dax, and D. Barna, Laser spectroscopy of pionic helium atoms, Nature (London) 581, 37 (2020).

[37] D. G. Fleming, D. M. Garner, L. C. Vaz, D. C. Walker, J. H. Brewer, and K. M. Crowe, Muonium chemistry-A review, in Positronium and Muonium Chemistry, edited by H. J. Ache, Advances in Chemistry Series Vol. 175 (American Chemical Society, Washington, DC, 1979), Chap. 13, pp. 279-334.

[38] M. Suffczyński, T. Kotowski, and L. Wolniewicz, Size of muonium hydride, Acta Phys. Pol., A 102, 351 (2002). 